\section{Validade de constructo da escala Experiences of Discrimination em uma população brasileira}

\author{
Validity of the Experiences of Discrimination scale \\ in a Brazilian population
}

\section{Resumo}

Um dos instrumentos mais utilizados na pesquisa epidemiológica sobre discriminação é o Experiences of Discrimination (EOD), utilizada no Brasil, porém não validado. O objetivo foi avaliar a confiabilidade e estrutura dimensional da escala EOD em uma população brasileira. Foi conduzido um estudo transversal com 1.380 adultos da cidade de Salvador, Bahia, Brasil. Realizou-se análise fatorial confirmatória (AFC) testando um modelo de dois fatores: experiência de discriminação e preocupação por discriminação. Os resultados da AFC mostraram parâmetros satisfatórios de ajuste, elevadas cargas fatoriais e valores adequados de confiabilidade, confirmando a consistência interna da escala. Identificaram-se correlações residuais envolvendo itens de ambos os fatores. A estrutura dimensional apresentada neste estudo destaca a importância de utilizar diferentes medidas de discriminação - interpessoais e grupais - que permitam em estudos futuros aprofundar nos efeitos do racismo sobre a saúde.
Gisel Lorena Fattore 1,2 Carlos Antonio Teles 3 Darci Neves dos Santos 2 Leticia Marques Santos 2 Michael Eduardo Reichenheim 4 Mauricio L. Barreto 2,5

\author{
1 Instituto de Salud Colectiva, \\ Universidad Nacional \\ de Lanús, Buenos Aires, \\ Argentina. \\ 2 Instituto de Saúde Coletiva \\ Universidade Federal da \\ Bahia, Salvador, Brasil. \\ 3 Universidade Estadual de \\ Feira de Santana, Feira de \\ Santana, Brasil. \\ 4 Instituto de Medicina \\ Social, Universidade do \\ Estado do Rio de Janeiro, Rio \\ de Janeiro, Brasil. \\ 5 Centro de Pesquisas \\ Gonçalo Muniz, Fundação \\ Oswaldo Cruz, Salvador, \\ Brasil.
}

\author{
Correspondência \\ G. L. Fattore \\ Instituto de Salud Colectiva, \\ Universidad Nacional de \\ Lanús. \\ 29 de Septiembre 3901 , \\ Buenos Aires 1826, \\ Argentina. \\ giselfattore@yahoo.com.ar
}

Racismo; Estudos de Validação; Análise Fatorial 


\section{Introdução}

A discriminação alude a todos os meios de expressão e institucionalização das relações sociais de dominação e opressão. Trata-se de um processo perante o qual um ou vários membros de grupos socialmente definidos são tratados de maneira diferente, e especialmente injusta, por causa de seu grupo social 1 .

O efeito da discriminação sobre a saúde é cada vez mais evidenciado, e hoje é reconhecido que contribui para as iniquidades em saúde, destacando-se o papel central do racismo na estruturação das desvantagens socioeconômicas dos grupos oprimidos 2,3.

No Brasil, as desigualdades raciais são ainda persistentes. Estatísticas oficiais mostram que a população que se autodeclara como parda ou preta, ou como indígena, se encontra em desvantagem em termos de recursos econômicos e acesso a oportunidades 4 , assim como em relação aos indicadores de saúde 5,6.

Os estudos epidemiológicos sobre o tema desenvolvidos nos países da América Latina e do Caribe mostram-se incipientes 7 . Uma análise bibliométrica da pesquisa sobre as desigualdades em saúde nos países da região evidenciou desinteresse no tocante à "raça"/cor da pele como tema de pesquisa, e pequeno número de estudos empíricos dedicados ao tema 7.

Almeida-Filho et al. 7 atribuíram o pouco desenvolvimento da área à existência de uma pretensa "democracia racial" que permeia o campo científico nesses países. De fato, durante grande parte do século XX a ideologia da miscigenação forjou a ideia de que o Brasil era um país sem linha de cor e consolidou o mito da democracia racial, segundo o qual não haveria na sociedade racismo nem discriminação ${ }^{8}$. Essa visão foi desafiada a partir dos anos 1950, quando sociólogos começaram a denunciar o racismo presente na sociedade brasileira 9,10. Uma primeira geração de pesquisadores colocou a ênfase no preconceito como mecanismo de reprodução e criação de desigualdades sociais, enquanto outros enfatizaram a estrutura de classes e as hierarquias sociais 8,10. Segundo Fry 11, a miscigenação outorga um valor positivo às relações sociais, favorecendo a integração e assimilação dos negros. No entanto, outros autores sustentam que a mestiçagem, ao impossibilitar a distinção racial, mascara o racismo presente na sociedade e impede sua superação 8,12 .

Embora diferentes formas de discriminação possam coexistir, alguns autores defendem a ideia de que a referência à "raça" se encontra subsumida em outras práticas discriminatórias 13, reconhecendo que as barreiras raciais se sobre- põem às de classe na manutenção das hierarquias raciais no país.

Como fenômeno complexo, a abordagem da discriminação tem desafios teóricos e metodológicos, e existe um amplo debate sobre as diferentes formas de medir o constructo. Grande parte dos instrumentos disponíveis utilizam indicadores de discriminação construídos mediante perguntas que avaliam percepções e experiências de discriminação entre os indivíduos ocorridas em diferentes situações do cotidiano.

Uns poucos instrumentos indagam sobre experiências de tratamento injusto vivenciadas por amigos, familiares ou membros de seu grupo social, desde que a discriminação não se limite às formas explícitas dirigidas a indivíduos particulares 14. A discriminação envolve também formas sutis, crenças e comportamentos simbólicos que denigram os indivíduos por causa de seu fenótipo ou grupo de filiação 15 . Segundo Krieger 14, as experiências que ocorrem na vida das pessoas próximas são fonte de preocupação e representam uma forma indireta de exposição ao racismo com consequências para a saúde. Além disso, os indicadores grupais oferecem uma medida auxiliar de discriminação que poderia detectar formas estruturais de racismo 16 . Exemplos desses instrumentos são a escala modificada Perceived Prejudice in Health Care 17,18, desenvolvida para estudar a discriminação em cuidados em saúde; e a Perceived Group Stigma Measure 19, desenhada para mensurar a percepção de estigma e discriminação sofrida por minorias sexuais.

Apesar da grande quantidade de instrumentos disponíveis para aferir discriminação ${ }^{20}$, grande parte deles carece de avaliações de validade e confiabilidade como base de endosso para uso em estudos populacionais 21 . Uma revisão recente revelou que, de um total de 24 instrumentos selecionados, 23 foram desenvolvidos nos Estados Unidos, apresentando escassas tentativas de adaptação transcultural. Tais instrumentos raramente foram escrutinados por pesquisadores independentes. Esse enfoque poderia contribuir no processo de validação dessas escalas, refutando ou confirmando os resultados obtidos originalmente 21 .

Várias perspectivas teóricas foram desenvolvidas para pensar as relações entre discriminação e os sistemas culturais nos quais acontecem. A perspectiva universalista defende que processos psicológicos são comuns a todos os seres humanos, mas a cultura influencia os comportamentos e a maneira como os conceitos se expressam 22. Em termos de equivalência, essa abordagem assume que os constructos não são iguais nas diversas culturas, portanto as particularidades de cada contexto precisam ser examinadas 
antes de se traduzir ou adaptar o instrumento 22 . O emprego de instrumentos padronizados é valorizado, uma vez que eles sejam submetidos a processos de adaptação transcultural 21 .

Um desses instrumentos, o Experiences of Discrimination (EOD), foi utilizado neste projeto. O EOD é bem consolidado nos Estados Unidos $23 \mathrm{e}$ ainda que tenha sido utilizado no Brasil recentemente 24 , não passou por processos de adaptação transcultural. Dentre os instrumentos disponíveis e amplamente utilizados em pesquisas sobre discriminação no momento de realização da pesquisa, o EOD foi escolhido por enfatizar a discriminação racial, considerando que ela é estruturante das desigualdades raciais no país.

\section{A escala EOD}

A primeira versão do EOD foi desenvolvida por Krieger em 1990 e utilizada no estudo Coronary Artery Risk Development in Young Adults (CARDIA) 25. Na referida versão, consta um conjunto de perguntas que indagam se os participantes já experimentaram discriminação, ou seja, se, em situações diversas, foram impedidos de fazer algo, incomodados ou inferiorizados, tendo por base diferenças de sexo, raça ou cor, posição socioeconômica, preferência sexual ou religião. As situações previstas foram sete: na escola, procurando emprego, no trabalho, ao comprar uma casa, nos cuidados médicos, na rua ou em estabelecimento público, pela polícia ou na justiça. Ademais, a escala contém dois itens que indagam sobre as respostas dos indivíduos ante o tratamento injusto.

Diferentemente da primeira versão, a escala revista por Krieger et al. 23, em 2005, e utilizada nesta pesquisa foca especificamente a experiência de eventos discriminatórios por causa racial. Aos itens que compunham a versão original, adicionaram-se dois novos: experiência de discriminação em lojas ou restaurantes, e ao solicitar empréstimo bancário. Além disso, incorporou-se um conjunto de itens que indagou sobre preocupação com questões raciais e questões globais. Os itens que compõem a experiência de discriminação foram validados em população americana por meio da análise fatorial confirmatória. Os resultados evidenciaram índices de ajuste de modelos adequados, com valores de Comparative Fit Index (CFI) de 0,966 e Root Mean Square Error of Aproximation (RMSEA) de 0,069 para o constructo experiência de discriminação, e alpha de Cronbach de $0,74{ }^{23}$. A escala mostrou associação de discriminação com sofrimento psicológico e tabagismo, e revelou alta correlação com outra escala de discriminação, a Everyday Discrimination Scale, desenvolvida por Williams et al. 26. Menor correlação foi encontrada quanto à frequência com que as pessoas se preocupam com a discriminação, indicando que esses itens estariam capturando um nível diferente de exposição à discriminação. Por essa razão, Krieger 14 tem destacado a importância de avaliar outras medidas de exposição ao racismo, como a exposição à discriminação do grupo, visto que a discriminação é uma experiência coletiva que as pessoas podem sofrer diretamente, mas também em grupo, além de sua própria experiência e consciência individual.

Dessa forma, o objetivo do presente trabalho é avaliar a confiabilidade e estrutura dimensional do EOD, incluindo na análise as medidas de exposição à discriminação do grupo, em uma amostra urbana de adultos da cidade de Salvador, Bahia, Brasil.

\section{Métodos}

\section{Desenho de estudo, população e área}

Trata-se de um estudo transversal que faz parte do Programa de Pesquisa SCAALA-Salvador. Os aspectos metodológicos do programa foram apresentados em outra publicação ${ }^{27}$. Sucintamente, a população de estudo foi integrada por 1.445 crianças participantes de uma coorte originalmente desenhada para estudar o impacto de um programa de saneamento ambiental sobre a saúde infantil no período de 1996 a 2004. A coleta de dados ocorreu desde janeiro até novembro de 2006, quando se realizaram visitas domiciliares por uma equipe previamente treinada e encarregada de aplicar o questionário de discriminação racial. Da amostra original envolvendo 1.445 crianças de 4 a 12 anos de idade e seus respectivos cuidadores, 1.380 aderiram ao presente estudo, obtendo-se uma taxa de resposta de $95 \%$. Os respondentes eram em sua maioria mães das crianças (75\%), e os demais eram avós, pais, irmãos ou vizinhos responsáveis pelo cuidado da criança.

\section{Instrumentos de mensuração utilizados no estudo}

\section{- A escala EOD no Brasil}

Com base nos procedimentos metodológicos propostos por Herdmann et al. ${ }^{28}$, iniciaram-se as primeiras etapas do processo de avaliação da equivalência transcultural para o EOD 29 , concebido em língua inglesa 23 .

Aderindo à perspectiva universalista citada anteriormente 22 , o processo originou-se de 
discussões entre os membros da equipe de coordenação da pesquisa, que incluiu epidemiologistas, psicólogos, especialistas na temática da saúde da população negra e indivíduos representativos da população-alvo. A finalidade foi determinar se a natureza e o significado dos constructos presentes no instrumento original (experiência de discriminação, preocupação com discriminação) e seus itens constituintes seriam pertinentes na população-alvo de Salvador. Aliás, procurouse identificar a existência de outras formas de discriminação na população brasileira, bem como a adequação desses constructos presentes na escala original para expressar o conceito discriminação. As discussões foram acompanhadas da revisão da literatura, colocando especial ênfase nas diferenças culturais e sócio-históricas entre as populações.

A versão em inglês do EOD foi submetida a processos de tradução e retrotradução no âmbito do Programa SCAALA. Dois membros do grupo de pesquisa com domínio da língua inglesa realizaram uma tradução preliminar da versão original para o português, a qual foi posteriormente aprimorada em discussões grupais com participação de expertos em inglês. Discutiu-se também a propriedade do significado dos termos nas retraduções em relação ao original. Sob a perspectiva do significado referencial dos termos constituintes, avaliou-se se o significado outorgado aos termos na versão original - em especial palavras-chave como discriminação e preocupação - correspondiam ao significado na respectiva tradução. Além do mais, e considerando que o instrumento captura conceitos culturalmente construídos, avaliou-se a correspondência entre percepção e impacto no respondente.

A versão final do instrumento foi submetida aos procedimentos de equivalência operacional. O processo de treinamento dos entrevistadores se deu em três etapas: inicialmente uma discussão teórica sobre o instrumento e o construto que ele pretende avaliar; em seguida, os entrevistadores fizeram aplicações-piloto de maneira individual e foram sanadas todas as dúvidas referentes à interpretação e ao processo de entrevista utilizando a escala. Por fim, os entrevistadores foram orientados a fazer aplicações em duplas distintas para consolidar e refinar o processo de coleta de dados com o instrumento. As palavras que não foram compreendidas pelos entrevistados durante essa fase foram listadas; em seguida, buscaram-se sinônimos em dicionários oficiais da língua portuguesa. Esses sinônimos eram utilizados em caso de não compreensão da pergunta.

Além disso, todos os entrevistadores passaram por um treinamento para estabelecimento e manutenção do Rapport, essencial para a confiança mútua durante a entrevista. O participante era orientado a responder de acordo com a sua experiência, destacando-se que não havia resposta certa ou errada. Os entrevistadores eram treinados para identificar casos de dúvidas do participante e responder a elas seguindo um procedimento padronizado. O estudo-piloto foi realizado em janeiro de 2006 com cuidadores de crianças atendidas em um centro comunitário de Salvador. Mesmo que as aplicações não tenham sido realizadas em número suficiente para um tratamento psicométrico dos dados, essa etapa permitiu realizar ajustes no questionário que finalmente foi aplicado à população participante do Programa SCAALA (Figura 1).

\section{Covariáveis}

Para caracterizar a amostra estudada, incluíramse as seguintes variáveis sociodemográficas: cor da pele autodeclarada, idade, sexo, renda média familiar e nível de instrução.

Com base em pesquisas que sugerem que a autoclassificação é o método que melhor reflete a construção das identidades raciais ${ }^{30}$, utilizouse a autodenominação dada pelo entrevistado para aferir a variável cor da pele. Obtiveram-se sete categorias: amarelo (28), branco (75), mulato (20), pardo (360), moreno (370), preto (517) e outros (9). Por causa do pequeno número de entrevistados para algumas das categorias obtidas, e considerando a concordância entre autoclassificação de cor/raça de acordo com pergunta aberta e fechada 30 , construíram-se três categorias: preto, branco e moreno/pardo, na qual foram incluídos aqueles autodenominados morenos, pardos, amarelos, mulatos e outros.

\section{Análise estatística}

Realizou-se a descrição do instrumento EOD pelo exame do padrão de resposta a todos os itens da escala. Com base em pesquisas anteriores $24 \mathrm{e}$ com a finalidade de obter categorias com maior frequência de respostas, os itens da escala foram dicotomizados em ausência de discriminação quando a resposta foi "nunca", e presença de discriminação, quando a resposta foi "pelo menos uma vez".

Com o intuito de avaliar a estrutura dimensional do instrumento, realizou-se uma análise fatorial confirmatória (AFC) ${ }^{31}$. Empregou-se o estimador robusto dos mínimos quadrados ponderados (WLSMV), que não assume a distribuição normal multivariada dos dados. Uma vez que os itens da escala foram utilizados de maneira dicotômica, utilizou-se a matriz de correlação 
Itens do questionário Experiences of Discrimination (EOD), versão traduzida ao português.

Resposta a tratamento injusto

A Se você sente que está sendo tratado injustamente, você geralmente:

Aceita isto como um fato da vida

Tenta fazer alguma coisa contra isto

B Se você está sendo tratado injustamente, você geralmente:

Fala com outras pessoas sobre isto

Guarda isto consigo mesmo

Perguntas sobre Preocupação

C Quando você era criança ou adolescente (menor de 18 anos), você se preocupava com as pessoas do seu grupo racial por serem tratadas injustamente por causa da raça ou cor da pele?

\section{Raramente ou nunca \\ Alguma parte do tempo \\ Todo o tempo}

D Quando você era criança ou adolescente (menor de 18 anos), você se preocupava por você sofrer experiências de tratamento injusto por causa da sua raça ou cor da pele?

Raramente ou nunca

Alguma parte do tempo

Todo o tempo

E No último ano você se preocupou com as pessoas do seu grupo racial por serem tratadas injustamente por causa da raça ou cor da pele?

\section{Raramente ou nunca \\ Alguma parte do tempo \\ Todo o tempo}

F No último ano você se preocupou por você sofrer experiências de tratamento injusto por causa da sua raça ou cor da pele?

\section{Raramente ou nunca \\ Alguma parte do tempo \\ Todo o tempo}

Experiência de discriminação por causa de raça/etnia ou cor da pele

Alguma vez sofreu a experiência de discriminação, sendo impedido de fazer alguma coisa, ou sentiu-se incomodado, ou levado a sentir-se inferior em algumas das seguintes situações por causa da sua raça, etnia ou cor? Quantas vezes isso aconteceu para cada situação seguinte? G Na escola

\section{Nunca}

Uma vez

Duas a três vezes

Mais de quatro vezes

H Ao procurar emprego

\section{Nunca}

Uma vez

Duas a três vezes

Mais de quatro vezes

No trabalho

\section{Nunca}

Uma vez

Duas a três vezes

Mais de quatro vezes
J Ao comprar uma casa

Nunca

Uma vez

Duas a três vezes

Mais de quatro vezes

K Ao procurar cuidados médicos

Nunca

Uma vez

Duas a três vezes

Mais de quatro vezes

L Em lojas ou restaurantes

Nunca

Uma vez

Duas a três vezes

Mais de quatro vezes

M Ao pedir crédito, empréstimo bancário

\section{Nunca \\ Uma vez}

Duas a três vezes

Mais de quatro vezes

N Na rua ou em estabelecimento público

$$
\text { Nunca }
$$

Uma vez

Duas a três vezes

Mais de quatro vezes

O Pela polícia ou no fórum

\section{Nunca}

Uma vez

Duas a três vezes

Mais de quatro vezes

Questões globais

P Com que frequência você sente que pessoas que não são brancas são discriminadas?

Nunca

Uma vez

Duas a três vezes

Mais de quatro vezes

Q Com que frequência você sente que você pessoalmente tem sido discriminado por causa da sua raça ou cor?

\section{Nunca \\ Uma vez}

Duas a três vezes

Mais de quatro vezes

R Alguma vez você prestou queixa por causa de discriminação racial?

Não

Sim 
tetracórica 31. A bondade de ajuste do modelo foi verificada mediante o CFI (valores maiores que 0,90 são considerados adequados), o TuckerLewis Index (TLI) (valores maiores que 0,95), e o RMSEA (valores menores que 0,05). Considerou-se, ainda, o intervalo de $90 \%$ de confiança (IC90\%) do RMSEA, que ofereceu suporte adicional para avaliar o ajuste do modelo (limite superior abaixo de 0,08 ) 31 . Para avaliar potenciais correlações residuais, foram utilizados os Índices de Modificação (IM), tendo em vista o ponto de corte de 10,0 a partir do qual se aceita a redundância de conteúdo em pares de itens.

Os procedimentos realizados com a escala dicotômica foram também aplicados à escala ordinal, obtendo-se resultados similares (dados não mostrados). A consistência interna foi avaliada pela Confiabilidade Composta (CC). A confiabilidade indica o grau de concordância entre um conjunto de indicadores de construtos latentes e suas respectivas mensurações. Valores aceitáveis são iguais ou superiores a 0,70 32. As validades convergente e discriminante foram avaliadas pela Variância Extraída (VME). AVME mede a quantidade da variância dos itens explicada por um fator, em relação à variância resultante de erros de mensuração dos itens componentes (valores $\geq$ 0,5 são considerados satisfatórios), sendo ainda indicativa da validade convergente do fator, ao sugerir que ao menos $50 \%$ da variância da medida se devem à hipótese subjacente. Assim, a validade fatorial discriminante é ratificada se a variância média de cada um dos fatores é maior do que o quadrado das correlações entre este fator e qualquer outro fator do modelo 33 . As análises descritivas foram realizadas no Stata versão 11 (StataCorp LP, College Station, Estados Unidos), e a análise fatorial, no programa Mplus 7.1 (Muthén \& Muthén, Los Angeles, Estados Unidos).

\section{Considerações éticas}

O protocolo utilizado neste estudo foi aprovado em 2004 pelo Comitê de Ética em Pesquisa do Instituto de Saúde Coletiva, Universidade Federal da Bahia e os participantes assinaram Termo de Consentimento Livre e Esclarecido.

\section{Resultados}

Dos 1.380 participantes, 95\% eram mulheres, um pouco mais da metade dos participantes $(53,4 \%)$ tinha entre 25 e 35 anos, 37,9\% identificaram-se como pretos, $56,7 \%$ morenos ou pardos e $5,4 \%$ brancos. Em relação à renda média familiar, $52 \%$ referiram receber menos que um salário mínimo por mês. Quase a metade $(47,5 \%)$ da população entrevistada referiu ter cursado entre o Ensino Fundamental incompleto e o Ensino Médio incompleto, $30,6 \%$ mencionaram ter completado o Ensino Médio ou mais, e $22 \%$ da população se declararam como analfabeta.

A Tabela 1 mostra a frequência de discriminação autorrelatada para todos os itens da escala. A respeito das perguntas sobre a preocupação com o tratamento injusto, a frequência com que os entrevistados afirmaram ter tal preocupação oscilou entre $30 \%$ e $40 \%$. No entanto, a frequência com que os entrevistados reportaram ter sofrido discriminação foi bem menor, variando de 7,9\% na busca de emprego até $1,1 \%$ ao comprar uma casa ou 1,1\% pela polícia ou pela Justiça.

\section{Análise fatorial confirmatória}

Na Tabela 2, observam-se os resultados da AFC. No Modelo A, os itens mostraram-se consistentes em ambos os fatores, experiência de discriminação e preocupação com discriminação, com valores maiores que 0,70 (valor de referência) na maioria das vezes. Os índices de adequação do modelo obtidos pela RMSEA, CFI e TLI encontraram-se dentro de patamares adequados. A correlação entre os fatores foi de 0,442 , e os valores de VME para os constructos foram maiores do que 0,50 , sugerindo que a variância em decorrência de erro de mensuração é menor do que a variância em razão do construto subjacente. Além disso, a VME mostrou valores maiores do que a estimativa quadrática de correlação entre os fatores $\left(0,4422^{2}=0,195\right)$, sugerindo que os itens individuais medidos representam bem suas respectivas dimensões latentes (fatores). ACC evidenciou valores maiores que 0,70 para ambos os fatores.

Apesar de os parâmetros de ajuste serem relativamente bons, a avaliação dos IM sugeriu cinco correlações residuais envolvendo os pares de itens $\mathrm{J} \leftrightarrow \mathrm{K}, \mathrm{L} \leftrightarrow \mathrm{M}, \mathrm{L} \leftrightarrow \mathrm{J}$ do fator Preocupação por discriminação, e os itens $\mathrm{B} \leftrightarrow \mathrm{F}, \mathrm{A} \leftrightarrow \mathrm{H}$ do fator experiências de discriminação. Visando corroborar as correlações residuais, partiu-se para uma reespecificação do modelo estimando livremente as correlações residuais 33 .

A Tabela 2 (Modelo B) apresenta os resultados da análise confirmatória após a inclusão dessas correlações residuais livremente estimadas. As cargas fatoriais permaneceram ao redor do valor de referência para a maior parte dos itens. Os parâmetros de ajuste melhoraram $(\mathrm{CFI}=0,998$, TLI = 0,998 e RMSEA = 0,010; IC90\%: 0,000-0,020) e são corroboradas as correlações residuais. Os resultados da variância extraída confirmaram a validade convergente e discriminante dos componentes da escala. Ademais, a CC mostrou 
Tabela 1

Frequência de discriminação autorrelatada segundo o questionário Experiences of Discrimination (EOD), escala com itens dicotômicos.

\begin{tabular}{|c|c|c|}
\hline Experiência de Discriminação & $\mathbf{n}$ & $\%$ \\
\hline \multicolumn{3}{|l|}{ Resposta ao tratamento injusto } \\
\hline \multicolumn{3}{|l|}{ A } \\
\hline \multicolumn{3}{|l|}{ Se você sente que está sendo tratado injustamente, você geralmente: } \\
\hline Aceita isto como um fato da vida & 581 & 41,8 \\
\hline Tenta fazer alguma coisa contra isto & 799 & 57,5 \\
\hline \multicolumn{3}{|l|}{ 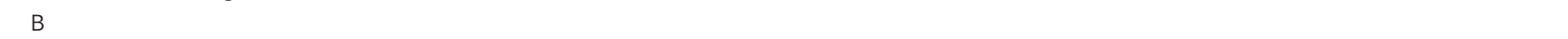 } \\
\hline \multicolumn{3}{|l|}{ Se você está sendo tratado injustamente, você geralmente: } \\
\hline Fala com outras pessoas sobre isto & 386 & 28,0 \\
\hline \multirow[t]{3}{*}{ Guarda isso consigo mesmo } & 994 & 72,0 \\
\hline & Nunca & $\begin{array}{l}\text { Uma vez ou } \\
\text { mais }\end{array}$ \\
\hline & n (\%) & $n(\%)$ \\
\hline \multicolumn{3}{|l|}{ Perguntas sobre preocupação } \\
\hline \multicolumn{3}{|l|}{ C } \\
\hline \multicolumn{3}{|l|}{ grupo racial por serem tratadas injustamente por causa da raça ou cor da pele? } \\
\hline \multicolumn{3}{|l|}{$\mathrm{D}$} \\
\hline $\begin{array}{l}\text { Quando você era criança ou adolescente (menor de } 18 \text { anos), você se preocupava por sofrer experiências de } \\
\text { tratamento injusto por causa da sua raça ou cor da pele? }\end{array}$ & $981(71,1)$ & $399(28,9)$ \\
\hline \multicolumn{3}{|l|}{ E } \\
\hline $\begin{array}{l}\text { No último ano, você se preocupou com as pessoas do seu grupo racial por serem tratadas injustamente por } \\
\text { causa da raça ou cor da pele? }\end{array}$ & $848(61,4)$ & $532(38,6)$ \\
\hline \multicolumn{3}{|l|}{$\mathrm{F}$} \\
\hline $\begin{array}{l}\text { No último ano, você se preocupou por sofrer experiências de tratamento injusto por causa da sua raça ou cor } \\
\text { da pele? }\end{array}$ & $974(70,6)$ & $406(29,4)$ \\
\hline \multicolumn{3}{|l|}{ Experiência de discriminação por causa da raça/etnia ou cor da pele } \\
\hline \multicolumn{3}{|l|}{ G } \\
\hline Na escola & $1.294(93,8)$ & $86(6,2)$ \\
\hline \multicolumn{3}{|l|}{$\mathrm{H}$} \\
\hline Ao procurar emprego & $1.270(92,1)$ & $109(7,9)$ \\
\hline \multicolumn{3}{|l|}{ l } \\
\hline No trabalho & $1.289(93,4)$ & $91(6,6)$ \\
\hline \multicolumn{3}{|l|}{$\mathrm{J}$} \\
\hline Ao comprar uma casa & $1.365(98,9)$ & $14(1,1)$ \\
\hline \multicolumn{3}{|l|}{$\mathrm{K}$} \\
\hline Ao procurar cuidados médicos & $1.311(95,0)$ & $69(5,0)$ \\
\hline \multicolumn{3}{|l|}{ L } \\
\hline Em lojas ou restaurantes & $1.304(94,5)$ & $76(5,5)$ \\
\hline \multicolumn{3}{|l|}{ M } \\
\hline Ao pedir crédito, empréstimo bancário & $1.362(98,7)$ & $18(1,3)$ \\
\hline \multicolumn{3}{|l|}{$\mathrm{N}$} \\
\hline Na rua ou em estabelecimento público & $1.276(92,5)$ & $104(7,5)$ \\
\hline \multicolumn{3}{|l|}{$\mathrm{O}$} \\
\hline Pela polícia ou no fórum & $1.365(98,9)$ & $15(1,1)$ \\
\hline \multicolumn{3}{|l|}{ Questões globais } \\
\hline \multicolumn{3}{|l|}{$\mathrm{P}$} \\
\hline Com que frequência você sente que pessoas que não são brancas são discriminadas? & $115(8,3)$ & $1.265(91,7)$ \\
\hline $\mathrm{Q}$ & & \\
\hline Com que frequência você sente que você pessoalmente tem sido discriminado por causa da sua raça ou cor? & $917(66,5)$ & $462(33,5)$ \\
\hline $\mathrm{R}$ & & \\
\hline Alguma vez você prestou queixa por causa de discriminação racial? & $1.373(99,5)$ & $7(0,51)$ \\
\hline
\end{tabular}


Tabela 2

Análise fatorial confirmatória para a versão traduzida da escala Experiences of Discrimination (EOD) com variáveis dicotômicas.

\begin{tabular}{|c|c|c|c|c|}
\hline \multirow[t]{2}{*}{ Itens } & \multicolumn{2}{|c|}{ Modelo A } & \multicolumn{2}{|c|}{ Modelo B } \\
\hline & Fator 1 * & Fator 2 ** & Fator 1 * & Fator 2 ** \\
\hline \multicolumn{5}{|l|}{ A } \\
\hline $\mathrm{Na}$ escola & 0,720 & - & 0,646 & - \\
\hline \multicolumn{5}{|l|}{ B } \\
\hline Ao procurar emprego & 0,910 & - & 0,819 & - \\
\hline \multicolumn{5}{|l|}{ C } \\
\hline No trabalho & 0,751 & - & 0,795 & - \\
\hline \multicolumn{5}{|l|}{ D } \\
\hline Ao comprar uma casa & 0,599 & - & 0,623 & - \\
\hline \multicolumn{5}{|l|}{$\mathrm{E}$} \\
\hline Ao procurar cuidados médicos & 0,681 & - & 0,712 & - \\
\hline \multicolumn{5}{|l|}{$\mathrm{F}$} \\
\hline Em lojas ou restaurantes & 0,916 & - & 0,809 & - \\
\hline \multicolumn{5}{|l|}{ G } \\
\hline Ao pedir crédito, empréstimo bancário & 0,793 & - & 0,835 & - \\
\hline \multicolumn{5}{|l|}{$\mathrm{H}$} \\
\hline Na rua ou em estabelecimento publico & 0,752 & - & 0,694 & - \\
\hline \multicolumn{5}{|l|}{ I } \\
\hline Pela polícia ou no fórum & 0,660 & - & 0,683 & - \\
\hline \multicolumn{5}{|l|}{ J } \\
\hline Preocupação com discriminação pelo grupo na infância & - & 0,718 & - & 0,546 \\
\hline \multicolumn{5}{|l|}{ K } \\
\hline Preocupação com discriminação consigo mesmo na infância & - & 0,785 & - & 0,764 \\
\hline \multicolumn{5}{|l|}{ L } \\
\hline Preocupação com discriminação pelo grupo no último ano & - & 0,897 & - & 0,681 \\
\hline \multicolumn{5}{|l|}{ M } \\
\hline Preocupação com discriminação consigo mesmo no último ano & - & 0,928 & - & 0,815 \\
\hline Correlação fatorial & \multicolumn{2}{|c|}{0,442} & \multicolumn{2}{|c|}{0,551} \\
\hline VME & 0,58 & 0,70 & 0,55 & 0,50 \\
\hline $\mathrm{CC}$ & 0,92 & 0,90 & 0,91 & 0,80 \\
\hline $\mathrm{CFI}$ & \multicolumn{2}{|c|}{0,963} & \multicolumn{2}{|c|}{0,998} \\
\hline TLI & \multicolumn{2}{|c|}{0,955} & \multicolumn{2}{|c|}{0,998} \\
\hline RMSEA (IC90\%) & \multicolumn{2}{|c|}{$0,046(0,040-0,052)$} & \multicolumn{2}{|c|}{$0,010(0,000-0,020)$} \\
\hline$J \leftrightarrow K$ & & & \multicolumn{2}{|c|}{0,600} \\
\hline$L \leftrightarrow M$ & & & \multicolumn{2}{|c|}{0,765} \\
\hline $\mathrm{L} \leftrightarrow \mathrm{J}$ & & & \multicolumn{2}{|c|}{0,319} \\
\hline $\mathrm{B} \leftrightarrow \mathrm{F}$ & & & & \\
\hline $\mathrm{A} \leftrightarrow \mathrm{H}$ & & & & \\
\hline
\end{tabular}

CC: Confiabilidade Composta; CFI: Comparative Fit Index; RMSEA (IC90\%): Root Mean Square Error of Aproximation (intervalo de 90\% de confiança);

TLI: Tucker-Lewis Index; VME: Variância Extraída.

* Experiências pessoais de discriminação;

** Preocupação com discriminação.

valores altos, sugerindo que todas as medidas representam de maneira consistente o mesmo constructo latente.

\section{Discussão}

Na avaliação da estrutura dimensional da escala EOD aplicada à população brasileira, o modelo de dois fatores - experiência de discriminação 
e preocupação com discriminação - apresentou parâmetros satisfatórios de ajuste na $\mathrm{AFC}$, os quais exibiram cargas fatoriais elevadas e valores adequados de confiabilidade, confirmando a consistência interna da escala. Esses achados são importantes já que revelam a capacidade do instrumento de capturar o constructo discriminação em um outro contexto, bastante distinto do contexto de desenvolvimento e validação.

Assim como no estudo original, todos os itens do fator experiência de discriminação permaneceram no modelo, evidenciando cargas fatoriais altas, especialmente nos domínios na procura de emprego e no trabalho, ou para pedir empréstimo bancário. Diferenças culturais e históricas devem ser levadas em conta na interpretação dos resultados. Vários autores têm destacado as particularidades da discriminação brasileira e as diferenças dela em relação à discriminação na sociedade norte-americana 8,11 . A intensa miscigenação que caracterizou as relações raciais no Brasil foi interpretada por alguns pesquisadores como prova da tolerância racial e da integração e assimilação dos pretos.

Porém, as relações raciais harmoniosas nunca deixaram de ser assimétricas. Segundo Sansone ${ }^{34}$, a discriminação no Brasil manifesta-se predominantemente em áreas consideradas "duras", em que a cor é um elemento relevante para a organização dos processos sociais. Desse modo, a discriminação expressar-se-ia mais intensamente naqueles âmbitos que envolvem relações de poder e competição entre os grupos raciais. Em outras formas de interação social, como as que se produzem em âmbitos de lazer, religiosos e nos relacionados com a cultura negra, as relações sociais mostrar-se-iam mais tolerantes e harmoniosas 11 .

Além disso, foram identificadas altas cargas fatoriais nos itens na rua e estabelecimentos públicos, e em lojas e restaurantes, ou na escola. Telles 8 tem salientado que o racismo consciente e explícito dirigido a indivíduos particulares e na forma de insultos ou injúrias raciais é a forma mais facilmente reconhecida do racismo no Brasil, apesar de outras formas sutis e indiretas de racismo, perpetuadas por mecanismos e práticas informais, persistirem e se naturalizarem na sociedade. Um exemplo é o racismo institucional que se observa nos cuidados em saúde, na escola e na justiça. Esses tipos de discriminação estão presentes na sociedade brasileira, como mostram as iniquidades no acesso, utilização de serviços e na atenção dispensada às mulheres pretas 35 ; as desvantagens que enfrentam as pessoas pretas no contato com o sistema de justiça e a polícia 36; e a persistência das desigualdades em matéria de educação 4 .
Cargas fatoriais menores se observam no item “ao comprar uma casa”, revelando uma vez mais discrepâncias com a sociedade norte-americana. A avaliação da discriminação ao comprar uma casa é pertinente nos Estados Unidos, onde os padrões de segregação dos pretos continuam a ser os mais altos entre todos os grupos étnico-raciais 37 . Pelo contrário, no Brasil não se verificam os níveis de discriminação institucional nem de segregação formal registrados nos Estados Unidos. Diante disso, um instrumento de aferição de discriminação desenvolvido no contexto brasileiro não contemplou tal item como parte dos domínios avaliados no questionário 38 .

Os itens que conformam o fator preocupação com discriminação mostraram cargas fatoriais altas, especialmente aqueles que indagam sobre a preocupação dos sujeitos de eles mesmos experimentarem discriminação. Embora o instrumento original tenha validado apenas os itens referentes às experiências diretas de discriminação, a utilização de outras medidas de exposição a racismo, como a percepção de discriminação para com outros, tem sido promovida por vários autores 26, inclusive Nancy Krieger 14 .

Como fenômeno complexo, o racismo não se expressa apenas nas formas de discriminação interpessoal, expressa-se também em formas institucionais, estruturais, e pela internalização da opressão. A literatura sobre estresse tem registrado que as experiências indiretas de estresse podem também afetar adversamente o indivíduo 14,39. Smith 40 tem destacado que a utilização de medidas grupais complementa a informação obtida no autorrelato de discriminação, ao oferecer uma medida da dimensão coletiva do racismo. Isso é importante no contexto brasileiro, no qual pretos são frequentemente vítimas diretas do racismo, ou espectadores de racismo contra membros de seu grupo racial. Nesse sentido, outros instrumentos mostraram estruturas fatoriais de mais de um fator, incorporando na validação subescalas referentes a diferentes dimensões do racismo. Como exemplos, vale citar o instrumento Measure of Indigenous Racism Experience desenvolvido por Paradies \& Cunningham 41, que avalia o sentimento de consciência racial com base na frequência com que as pessoas pensam sobre ser indígena; ou Perceived Ethnic Discrimination Questionnaire, que avalia a exposição à discriminação de amigos ou membros da família do respondente 18. Outra escala, a Negative Life Events Scale, indaga especificamente sobre a preocupação do respondente ou familiar próximo com certos eventos estressantes na vida, entre eles o racismo e a discriminação ${ }^{42}$.

Concordante com outros estudos 43,44, no presente estudo, os indivíduos apresentaram 
tendência a perceber experiências de discriminação mais frequentemente relacionadas com seu grupo do que com a sua pessoa. Contudo, as respostas que as pessoas reportam para si mesmas e para o seu grupo não são diretamente comparáveis por duas razões. Primeiro, porque as pessoas podem ter preocupações com os outros integrantes de seu grupo de forma mais intensa do que consigo mesmas em razão do que elas pessoalmente tenham experimentado. Segundo, porque existe um problema chamado de "discrepância de discriminação entre a pessoa e o grupo" (personal/group discrimination discrepancy - PGDD), um fenômeno conhecido na psicologia que sugere que as pessoas tendem a sub-relatar suas próprias experiências de discriminação 14 .

Também se aponta a tendência dos entrevistados a fornecer respostas socialmente desejáveis, ou desenvolver mecanismos protetores, os quais contribuiriam para a subestimativa do relato 14 . Ainda é possível que as perguntas se centrem em diferentes tipos de informação, talvez, as perguntas "do grupo" capturem sentimentos de identidade grupal, ao passo que as perguntas "individuais" evocam aspectos pessoais da identidade ${ }^{43}$. Ser membro de um grupo desfavorecido produz uma identidade com os sentimentos e atributos do grupo étnico-racial; por outro lado, a negação das experiências pessoais de discriminação seria o resultado do distanciamento das pessoas dos atributos negativos do grupo ao qual pertencem 44 .

O efeito do PGDD tem sido encontrado em medidas de autoestima, identidade, atitudes e estereótipos, medidas que questionam se esses mecanismos autoprotetores alcançam a consciência e são reconhecidos pelo sujeito. Desse modo, alguns pesquisadores têm investigado se esse efeito está ligado a um modo especial de medição da exposição à discriminação, e desenvolvido outros instrumentos menos sensíveis ao PGDD 45.

Do mesmo modo que na população norteamericana, aqui também se verificaram correlações residuais. Elas ocorreram entre os itens discriminação na escola e em estabelecimentos públicos, e entre os itens procurando emprego e em lojas ou restaurantes do Fator 1; e entre todos os itens que conformam o Fator 2. A presença de correlações residuais dentro de um mesmo fator é indicativa de redundância semântica, decorrente da utilização de redação semelhante para expressar ideias diferentes.

De uma perspectiva teórica, os itens redundantes do Fator 1 manifestariam a memória de eventos discriminatórios no âmbito público, como as escolas e outros estabelecimentos públicos $(\mathrm{A} \leftrightarrow \mathrm{H})$; e a lembrança de experiência de discriminação relacionada com a aparência física, como na demanda serviços e procura de emprego $(\mathrm{B} \leftrightarrow \mathrm{F})$. Já o conteúdo redundante do Fator 2 expressa a memória de discriminação no passado $(\mathrm{J} \leftrightarrow \mathrm{K})$; memória de discriminação no presente $(\mathrm{L} \leftrightarrow \mathrm{M})$; e memória de discriminação para com membros do grupo racial $(\mathrm{L} \leftrightarrow \mathrm{J})$. Da mesma forma que no estudo americano, houve correlações residuais com ajustes aceitáveis.

A análise da confiabilidade mostrou adequada convergência dos itens dentro de cada fator, indicando que os significados dos mesmos respondem ao mesmo constructo. Igualmente, os fatores mostraram validade discriminante, permitindo diferenciar um constructo de outro. Desse modo, tais medidas estariam capturando diferentes aspectos da discriminação dos indivíduos para consigo mesmos e como membros de um grupo social.

Esses resultados enfatizam a importância de discernir se a utilização de apenas o autorrelato de discriminação é suficiente para estudar a discriminação. Independentemente de qualquer experiência real, a preocupação informa sobre a consciência da discriminação na sociedade e o sentimento de vulnerabilidade ante os atos discriminatórios. Nazroo ${ }^{16}$ reconhece outra perspectiva de viver em uma sociedade racista, além da experiência individual de discriminação. Ao comparar os grupos dominantes com os grupos subordinados, membros do último reconheceriam a própria situação de desvantagem e exclusão. Williams \& Mohammed 39 destacam que avaliar a exposição à discriminação racial em toda a sua complexidade também requer capturar os efeitos da discriminação sobre os outros, bem como os potenciais efeitos intergeracionais do racismo.

Este estudo evidencia algumas limitações. A população de estudo foi constituída por indivíduos predominantemente pobres do Nordeste do Brasil, a maioria mulheres, e é perceptível que pardos e pretos estiveram sobrerrepresentados na amostra. O significado do racismo para essa população pode ser diferente do significado para populações de outras regiões do país, onde as relações interpessoais e os níveis de desigualdade são diferentes.

Aliás, o Brasil é um país de dimensões continentais, com diversidade nos níveis socioeconômicos e na composição étnica da população. A cidade de Salvador apresenta o maior índice de isolamento espacial de pretos do Brasil e alta proporção de população autodeclarada preta 8 . Adicionalmente, o Estado da Bahia teve um papel central na história da escravidão, e continua a ter níveis marcantes de discriminação racial 
estruturantes das desvantagens cumulativas da população negra 46. Logo, devemos ressaltar que os bons parâmetros de ajuste obtidos em nossa amostra podem refletir as particularidades sociais e históricas dessa população e não ser reproduzíveis em outros conjuntos populacionais do país.

Ainda que o modelo aparente ajustar-se relativamente bem, detectaram-se várias correlações residuais indicativas de violação de independência local. Possivelmente, este fato se relaciona com a incompletude do processo de adaptação transcultural do instrumento. Também é possível que existam erros no desenho e formulação das perguntas, uma vez que essas correlações já estiveram presentes na validação do instrumento original. Ainda assim, optou-se por conservar o total das perguntas como foram indicadas na proposta original, já que essa é a primeira iniciativa de adaptação transcultural do instrumento em população brasileira de que se tem notícia. Sendo assim, estudos adicionais em outras populações são necessários.

Uma vez corroborada a redundância de conteúdo, é possível sugerir algumas alternativas para o uso do instrumento em população brasileira. Uma solução é a utilização do instrumento no seu estado atual, considerando as correlações residuais, mas em modelos de equações estruturais nos quais o componente do erro de medida pudesse ser plenamente especificado. Outras soluções a explorar envolvem: (1) avaliação escalar dos itens redundantes, remover parcimoniosamente um dos dois itens de cada par; (2) por par, substituir um dos itens por outro "melhor", não redundante; (3) juntar o conteúdo semântico dos itens correlacionados em uma única questão, visando capturar as informações de ambos os itens originais 33 .

Na perspectiva confirmatória, outras análises podem ser feitas com o EOD. De importância para populações heterogêneas é a avaliação da invariância dos parâmetros estimados no modelo, de modo a estabelecer se as propriedades das medidas são equivalentes nos subgrupos populacionais 31 . Essa análise permitiria afirmar com maior segurança se o constructo subjacente "discriminação" é comparável nos grupos de cor. Outras análises envolvendo o modo como os itens mapeiam o traço latente em termos de intensidade seriam recomendadas 33 .

Apesar dos problemas identificados nessa escala, este estudo contribui para a compreensão do racismo como fenômeno multidimensional. Não existe uma metodologia padronizada para aferir a discriminação, e os instrumentos disponíveis para aplicar à população brasileira são escassos. No Brasil, as desigualdades raciais são marcantes, e a discriminação racial é visível em muitas esferas da vida social. Tendo em vista o impacto desses fatos sobre a saúde, existe a necessidade de avaliar de maneira sistemática a persistência de discriminação na população e o modo como afeta a saúde dos grupos étnico-raciais para orientar políticas destinadas à redução das desigualdades sociais.

\section{Conclusões}

Os resultados obtidos nesta pesquisa contribuem para a avaliação da discriminação racial no contexto brasileiro ao iniciar o processo de adaptação transcultural do instrumento EOD que foi aplicado em um estudo de base populacional.

A estrutura dimensional apresentada neste estudo destaca a importância de serem usadas diferentes medidas de discriminação - interpessoais e grupais -, que representam aspectos diferentes da visão que os indivíduos têm de si mesmos e em relação aos sentimentos e atributos dos grupos aos quais pertencem.

As diferenças encontradas em relação ao estudo original ressaltam a necessidade de validar os indicadores de discriminação racial em contextos distintos do de origem, incorporando múltiplas medidas de discriminação racial que permitam em estudos futuros aprofundar o conhecimento dos efeitos do racismo sobre a saúde. Os problemas identificados nesta análise apontam a necessidade de aperfeiçoar o instrumento, a fim de melhorar sua qualidade psicométrica e dar continuidade ao processo de adaptação transcultural iniciado com esta pesquisa.

Aliás, recomenda-se fortemente a realização de novos estudos que envolvam outras populações brasileiras, visando fortalecer as investigações sobre a discriminação racial no país. 


\section{Colaboradores}

G. L. Fattore contribuiu na formulação da pergunta de investigação, realizou a revisão bibliográfica, análise dos dados e redação das diversas versões do artigo. C. Teles, L. M. Santos e M. E. Reichenheim contribuíram na análise, interpretação dos dados, escrita e revisão crítica do artigo. D. N. Santos coordenou o processo de tradução e adaptação da escala EOD, elaborando a versão final em português e aplicação na população do estudo. M. L. Barreto colaborou na elaboração do desenho de estudo, interpretação dos dados, leitura e edição do manuscrito. Todos os autores participaram da elaboração da versão final do artigo.

\section{Referências}

1. Karlsen S, Nazroo JY. Measuring and analyzing "race", racism, and racial discrimination. In: Oakes M, Kaufman JS, editors. Methods in social epidemiology. San Fransisco: Wiley Press; 2006. p. 86111.

2. Krieger N. Does racism harm health? Did child abuse exist before 1962? On explicit questions, critical science, and current controversies: an ecosocial perspective. Am J Public Health 2003; 93:194-9.

3. Nazroo JY. The structuring of ethnic inequalities in health: economic position, racial discrimination, and racism. Am J Public Health 2003; 93:277-84.

4. Instituto de Pesquisa Econômica Aplicada. Desigualdades raciais, racismo e políticas públicas: 120 anos após a abolição. Brasília: Instituto de Pesquisa Econômica Aplicada; 2008.

5. Chor D, Lima CRA. Aspectos epidemiológicos das desigualdades raciais em saúde no Brasil. Cad Saúde Pública 2005; 21:1586-94.

6. Martins AL. Mortalidade materna de mulheres negras no Brasil. Cad Saúde Pública 2006; 22:2473-9.

7. Almeida Filho N, Kawachi I, Filho AP, Dachs JN. Research on health inequalities in Latin America and the Caribbean: bibliometric analysis (1971-2000) and descriptive content analysis (1971-1995). Am J Public Health 2003; 93:2037-43.

\section{Agradecimentos}

Agradecemos à pesquisadora Edna Araujo pela interlocução sobre a propriedade das questões traduzidas, considerando sua experiência de investigação epidemiológica com a população negra.
8. Telles E. O significado da raça na sociedade brasileira. 2004. http://disciplinas.stoa.usp.br/pluginfi le.php/386261/mod_resource/content/2/livro-OSignificado-da-Raca-na-Sociedade-Brasileira.pdf (acessado em Abr/2013).

9. Guimarães ASA. Preconceito de cor e racismo no Brasil. Rev Antropol (São Paulo) 2004; 47:9-43.

10. Maio MC. O contraponto paulista: Florestan Fernandes, Oracy Nogueira e o Projeto Unesco de relações raciais. Antíteses 2014; 7:10-39.

11. Fry PH. A persistência da raça: ensaios antropológicos sobre o Brasil e a África Austral. Rio de Janeiro: Editora Civilização Brasileira; 2005.

12. Guimarães ASA. Entrevista com Carlos Hasenbalg. Tempo Social 2006; 18:259-68.

13. Osorio RG. A mobilidade social dos negros brasileiros. Brasília: Instituto de Pesquisa Econômica Aplicada; 2004. (Texto para Discussão, 1033).

14. Krieger N. Embodying inequality: a review of concepts, measures, and methods for studying health consequences of discrimination. Int J Health Services 1999; 29:295-352.

15. Stuber J, Meyer I, Link B. Stigma, prejudice, discrimination and health. Soc Sci Med 2008; 67: 351-7. 
16. Nazroo JY. Genetic, cultural or socio-economic vulnerability? Explaining ethnic inequalities in Health. Sociol Health Illn 1998; 20:710-30.

17. Kressin NR, Kristal RL, Manze M. Perceptions of race/ethnicity based discrimination: a review of measures and evaluation of their usefullness for the health care setting. J Health Care Poor Underserved 2008; 19:697-730.

18. Brondolo E, Kelly KP, Coakley TG, Gordon T, Thompson S, Levy E. The Perceived Ethnic Discrimination Questionnaire: development and preliminary validation of a community version. J Appl Soc Psychol 2005; 35:335-65.

19. McGarrity LA, Huebner DM, McKinnon RK. Putting stigma in context: do perceptions of group stigma interact with personally experienced discrimination to predict mental health? Group Process Intergroup Relat 2013; XX:1-15.

20. Paradies Y. A systematic review of empirical research on self-reported racism and health. Int J Epidemiol 2006; 35:888-901.

21. Bastos JL, Celeste RK, Faerstein E, Barros L. Discriminação racial e saúde: uma revisão sistemática de escalas com foco em suas propriedades psicométricas. Saúde Transform Soc 2011; 1: 4-16.

22. Herdman M, Fox-Rushby J, Badia X. "Equivalence" and the translation and adaptation of health-related quality of life questionnaires. Qual Life Res 1997; 6:237-47.

23. Krieger N, Smith K, Naishadham D, Hartman C, Barbeau EM. Experiences of discrimination: validity and reliability of a self-report measure for population health research on racism and health. Soc Sci Med 2005; 61:1576-96.

24. Pavão ALB, Ploubidis GB, Werneck G, Campos MR. Racial discrimination and health in Brazil: evidence from a population-based survey. Ethn Dis 2012; 22:353-9.

25. Krieger N, Sidney S. Racial discrimination and blood pressure: the CARDIA study of young black and white adults. Am J Public Health 1996; 86:1370-8.

26. Williams DR, Yu Y, Jackson JS, Anderson NB. Racial differences in physical and mental health: socio-economic status, stress and discrimination. J Health Psychol 1997; 2:335-51.

27. Barreto ML, Cunha SS, Alcântara-Neves N, Carvalho LP, Cruz AA, Stein RJ, et al. Risk factors and immunological pathways for asthma and other allergic diseases in children: background and methodology of a longitudinal study in a large urban center in Northeastern Brazil (Salvador-SCAALA study). BMC Pulm Med 2006; 6:15.

28. Herdman M, Fox-Rushby J, Badia X. A model of equivalence in a cultural adaptation of HRQoL instruments: the universalist approach. Qual Life Res 1998; 7:323-35.

29. Reichenheim ME, Moraes CL. Operacionalização de adaptação transcultural de instrumentos de aferição usados em epidemiologia. Rev Saúde Pública 2007; 41:665-73.

30. Maio MC, Monteiro S, Chor D, Faerstein E, Lopes C. Cor/raça no Estudo Pró-Saúde: resultados comparativos de dois métodos de autoclassificação no Rio de Janeiro, Brasil. Cad Saúde Pública 2005; 21:171-80
31. Brown TA. Confirmatory factor analysis for applied research. 2nd Ed. New York: The Guilford Press; 2015.

32. Hair JF, Black WC, Babin BJ, Anderson RE, Tatham RL. Análise multivariada dos dados. 6a Ed. Porto Alegre: Bookman; 2009.

33. Reichenheim ME, Hökerberg YHM, Moraes CL. Assessing construct structural validity of epidemiological measurement tools: a seven-step roadmap. Cad Saúde Pública 2014; 30:927-39.

34. Sansone L. As relações raciais em Casa-Grande \& Senzala revisitadas à luz do processo de internacionalização e globalização. In: Maio MC, Santos RV, organizadores. Raça, ciência e sociedade. Rio de Janeiro: Editora Fiocruz/CCBB; 2006. p. 207-18.

35. Leal MC, Gama SGN, Cunha CB. Racial, sociodemographic, and prenatal and childbirth care inequalities in Brazil, 1999-2001. Rev Saúde Pública 2005; 39:100-7.

36. Santos SA. Who is black in Brazil? A timely or a false question in Brazilian race relations in the era of affirmative action? Latin Am Perspect 2006; 33:30-48.

37. Massey DS, Rothwell J, Domina T. The changing bases of segregation in the United States. Ann Am Acad Pol Soc Sci 2009; 626:10.1177/0002716209343558.

38. Bastos JL, Faerstein E, Celeste RK, Barros AJD. Explicit discrimination and health: development and psychometric properties of an assessment instrument. Rev Saúde Pública 2012; 46:269-78.

39. Williams DR, Mohammed SA. Discrimination and racial disparities in health: evidence and needed research. J Behav Med 2009; 32:20-47.

40. Smith T. Measuring racial and ethnic discrimination. Chicago: National Opinion Research Center; 2002. (GSS Methodological Report, 96).

41. Paradies YC, Cunningham J. Development and validation of the Measure of Indigenous Racism Experiences (MIRE). Int J Equity Health 2008; 7:9.

42. Kowal E, Gunthorpe W, Bailie RS. Measuring emotional and social wellbeing in Aboriginal and Torres Strait Islander populations: an analysis of a Negative Life Events Scale. Int J Equity Health 2007; 6:18.

43. Taylor DM, Wright SC, Moghaddam FM, Lalonde $\mathrm{RN}$. The personal/group discrimination discrepancy. Pers Soc Psychol Bull 1990; 16:254-62.

44. Carney DR, Banaji MR, Krieger N. Implicit measures reveal evidence of personal discrimination. Self Identity 2010; doi:10.1080/13594320902847927.

45. Krieger N, Carney D, Lancaster K, Waterman PD, Kosheleva A, Banaji M. Combining explicit and implicit measures of racial discrimination in health research. Am J Public Health 2010; 100:1485-92.

46. Garcia AS. Desigualdades raciais e segregação urbana contemporâneas: Salvador, cidade d'Oxum e Rio de Janeiro, cidade de Ogum [Tese de Doutorado]. Rio de Janeiro: Instituto de Pesquisa e Planejamento Urbano, Universidade Federal do Rio de Janeiro; 2006 


\section{Abstract}

One of the most widely used tools in epidemiological research on discrimination is the Experiences of Discrimination (EOD) scale, used but not validated in Brazil. The objective was to assess the reliability and dimensional structure of the EOD scale in a Brazilian population. A cross-sectional study was performed with 1,380 adults in the city of Salvador, Bahia State, Brazil. Confirmatory factor analysis (CFA) was performed testing a two-factor model: experiences of discrimination and concerned about discrimination. The results of CFA showed satisfactory fit, high factor loads, and adequate reliability, confirming the scale's internal consistency. Residual correlations were identified involving items from both factors. The dimensional structure presented in this study highlights the importance of using different measures of discrimination (interpersonal and group) to allow more in-depth future research on the effects of racism on health.

Racism; Validation Studies; Statistical Factor Analysis

\section{Resumen}

Uno de los instrumentos más utilizados en investigación epidemiológica sobre discriminación es el Experiences of Discrimination (EOD), utilizado en Brasil, aunque aún no fue validado em esta población. El objetivo de este trabajo fue evaluar la validez y estructura dimensional de la EOD en una población brasileña. Se realizó un estudio transversal con 1.380 adultos de la ciudad de Salvador, Bahía, Brasil. Se realizó un análisis factorial confirmatorio (AFC), probando un modelo de dos factores: experiencias de discriminación y preocupación por la discriminación. Los resultados de la AFC mostraron parámetros satisfactorios de ajuste, elevadas cargas factoriales y valores adecuados de confiabilidad, confirmando la consistencia interna de la escala. Se identificaron correlaciones residuales, involucran do ítems de ambos factores. La estructura dimensional presentada en este estudio destaca la importancia de utilizar diferentes medidas de discriminacióninterpersonales y grupales-, que permitan en estudios futuros profundizar en los efectos del racismo sobre la salud.

Racismo; Estudios de Validación; Análisis Factorial
Recebido em 27/Jun/2015

Versão final reapresentada em 29/Out/2015 Aprovado em 16/Nov/2015 